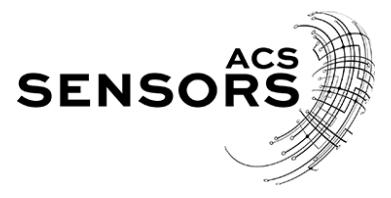

Subscriber access provided by Caltech Library

\title{
Letter
}

\section{SERS-Based Label-Free Insulin Detection at Physiological Concentrations for Analysis of Islet Performance}

Hyunjun Cho, Shailabh Kumar, Daejong Yang, Sagar R. Vaidyanathan, Kelly

Woo, Ian Garcia, Hao Jan Shue, Youngzoon Yoon, Kevin Ferreri, and Hyuck Choo

ACS Sens., Just Accepted Manuscript • DOI: 10.1021/acssensors.7b00864 • Publication Date (Web): 11 Jan 2018

Downloaded from http://pubs.acs.org on January 12, 2018

\section{Just Accepted}

"Just Accepted" manuscripts have been peer-reviewed and accepted for publication. They are posted online prior to technical editing, formatting for publication and author proofing. The American Chemical Society provides "Just Accepted" as a free service to the research community to expedite the dissemination of scientific material as soon as possible after acceptance. "Just Accepted" manuscripts appear in full in PDF format accompanied by an HTML abstract. "Just Accepted" manuscripts have been fully peer reviewed, but should not be considered the official version of record. They are accessible to all readers and citable by the Digital Object Identifier (DOI®). "Just Accepted" is an optional service offered to authors. Therefore, the "Just Accepted" Web site may not include all articles that will be published in the journal. After a manuscript is technically edited and formatted, it will be removed from the "Just Accepted" Web site and published as an ASAP article. Note that technical editing may introduce minor changes to the manuscript text and/or graphics which could affect content, and all legal disclaimers and ethical guidelines that apply to the journal pertain. ACS cannot be held responsible for errors or consequences arising from the use of information contained in these "Just Accepted" manuscripts. 


\title{
SERS-Based Label-Free Insulin Detection at Physiological Concentrations for Analysis of Islet Performance
}

\author{
Hyunjun Cho ${ }^{1, \dagger}$, Shailabh Kumar ${ }^{2, \dagger}$, Daejong Yang ${ }^{2}$, Sagar Vaidyanathan ${ }^{1}$, Kelly Woo ${ }^{1}$, Ian \\ Garcia $^{1}$, Hao J. Shue ${ }^{1}$, Youngzoon Yoon ${ }^{3}$, Kevin Ferreri ${ }^{4}$, Hyuck Choo $^{*, 1,2}$ \\ ${ }^{1}$ Department of Electrical Engineering, ${ }^{2}$ Department of Medical Engineering, \\ California Institute of Technology, Pasadena, CA 91125, United States \\ ${ }^{3}$ Device Lab, Device \& System Research Center, Samsung Advanced Institute of Technology \\ (SAIT), Suwon, 16678, Republic of Korea \\ ${ }^{4}$ Department of Translational Research and Cellular Therapeutics, Diabetes and Metabolism \\ Research Institute, Beckman Research Institute of the City of Hope, Duarte, CA
}

\author{
$\dagger$ These authors contributed equally \\ Corresponding author*E-mail: hchoo@,caltech.edu
}




\begin{abstract}
Label-free optical detection of insulin would allow in vitro assessment of pancreatic cell functions in their natural state and expedite diabetes-related clinical research and treatment, however no existing method has met these criteria at physiological concentrations. Using spatially-uniform 3D gold-nanoparticle sensors, we have demonstrated surface-enhanced Raman sensing of insulin in the secretions from human pancreatic islets under low and high glucose environments without the use of labels such as antibodies or aptamers. Label-free measurements of the islet secretions showed excellent correlation among the ambient glucose levels, secreted insulin concentrations, and measured Raman-emission intensities. When excited at $785 \mathrm{~nm}$, plasmonic hotspots of the densely-arranged 3D gold-nanoparticle pillars as well as strong interaction between sulphide linkages of the insulin molecules and the gold nanoparticles produced highly sensitive and reliable insulin measurements down to $100 \mathrm{pM}$. The sensors exhibited a dynamic range of $100 \mathrm{pM}$ to $50 \mathrm{nM}$ with an estimated detection limit of $35 \mathrm{pM}$, which covers the reported concentration range of insulin observed in pancreatic cell secretions. The sensitivity of this approach is approximately four orders of magnitude greater than previously reported results using label-free optical approaches, and it is much more costeffective than immunoassay-based insulin detection widely used in clinics and laboratories. These promising results may open up new opportunities for insulin sensing in research and clinical applications.
\end{abstract}

\title{
Keywords: SERS, Insulin, biosensing, plasmonics, nanoparticles
}


Hormones are chemical messengers that control a wide variety of functions in the human body. Maintaining adequate hormone levels is extremely important for human health and disruption to these levels can result in life-debilitating conditions. Simple and easy measurements of hormonal secretions ex vivo or in vivo are essential for implementing next generation biosensors, allowing convenient monitoring of health and early disease detection.

One of the most prevalent diseases resulting from hormonal dysfunction is diabetes, which arises from a disruption in the release of insulin in the body. ${ }^{1-2}$ Insulin is a peptide hormone which is secreted by beta cells, one of five primary cell-types which populate pancreatic cellular clusters known as islets. The concentration of insulin secreted from beta cells in plasma has been reported to vary between $100 \mathrm{pM}$ (fasting) and $2 \mathrm{nM}$ (about 1 hour after glucose intake) in non-diabetic individuals. ${ }^{3-4}$ In diabetic individuals, functional damage to the beta cells reduces or inhibits their ability to release insulin. One of the leading methodologies for treatment of type-1 diabetes is pancreatic islet transplantation, where healthy islets harvested from deceased donors are transplanted into diabetic patients. ${ }^{5-6}$ Since the number of donors is limited, methodologies that can improve the efficiency and success of the transplantation process are urgently needed. Before transplantation these islets are screened for their viability and functional response to changing glucose concentration in order to reduce the chances of transplant failure..$^{7-9}$ Sensors capable of detecting secretion of insulin from beta cells in a costeffective, label-free manner with minimal stress to the cells can serve vital roles in clinical quality assessment of islets. External monitoring of insulin concentration can also help in disease diagnosis and management as well as prevent potentially fatal insulin overdoses or hypoglycemia in diabetic patients. ${ }^{10}$ 
Various sensing methods have been previously explored for insulin detection including radioimmunoassays, ${ }^{11}$ mass-spectrometry, ${ }^{12}$ photoluminescence, ${ }^{13}$ electrochemical methods, ${ }^{14}$ electrophoresis-dependent immunoassays, ${ }^{15-16}$ surface plasmon-resonance (SPR)-based competitive binding assays, ${ }^{17}$ and fluorescence resonance energy transfer (FRET). ${ }^{18}$ The optical detection techniques such as SPR, photoluminescence, and FRET suffer from poor sensitivity and cannot detect insulin at physiologically observed picomolar concentrations. ${ }^{13,}$ 17-18 Electrochemical impedance spectroscopy and immunoassays have reported more sensitive insulin-specific detection, however these methods have primarily relied on capture agents such as antibodies for detection. ${ }^{11,14,19}$ These antibodies are not only expensive, but have also been shown to disrupt the natural behavior of live cells. ${ }^{20-21}$

For applications such as pancreatic islet transplantation, a label-free, optical sensing method capable of performing ultrasensitive detection of insulin without using capturing agents can help improve the cost-effectiveness as well help the islet cells remain as close to their natural state as possible during the pre-surgical screening process. Surface-enhanced Raman spectroscopy (SERS) is an ideal approach for optical label-free sensing because it identifies targeted molecules based on their unique vibrational and rotational signatures. ${ }^{22-26}$ Application of SERS for hormone detection appears relatively unexplored due to minimal experimental success: previously reported SERS-based quantitative insulin sensors were limited due to weaklyenhancing substrates made of randomly dispersed nanoparticles resulting in micromolar detection sensitivity, approximately 2 to 4 orders of magnitude larger than clinically-relevant insulin levels. $^{27-30}$

In this study, we report highly sensitive SERS-based insulin sensing at clinically relevant concentrations using a non-resonant SERS substrate with strong signal enhancement and wafer- 
scale uniformity. ${ }^{31-34}$ The sensors were batch-fabricated using a simple two-step process that produced 3 dimensional (3D) gold-nanoparticle (Au-NP) clusters packed densely in a vertical, pillar-like arrangement. ${ }^{33}$ The 3D pillar-like geometry of the clusters adsorb and excite insulin molecules with vertically packed plasmonic hotspots uniformly over a large surface area for enhanced Raman emissions. This geometry provides significantly stronger and more spatially uniform enhancement than previously used substrates and greatly improved the detection limit. ${ }^{31-}$ ${ }^{36}$ The obtained result is several orders of magnitude better than previously reported values produced using SERS approaches, ${ }^{27-30}$ and the detection limit of the substrate is estimated as 35 pM. Using our method, we characterized the insulin concentrations in pancreatic islet secretions that were collected under low and high glucose conditions. The measurements obtained using our approach showed excellent consistency and paralleled the concentrations obtained using an enzyme-linked immunosorbent assay (ELISA).

The fabrication of the SERS substrate was described in detail by Yang et al. ${ }^{33} \mathrm{~A}$ summary of the fabrication has been provided in the Supporting Information (Figure S1). The cross-sectional view of the 3D Au-NP SERS substrate acquired using scanning electron microscopy (SEM) is shown in Figure 1a. The SEM image reveals a dense, vertical arrangement of gold nanoparticle clusters that, based on experimental results, provide plasmonic hotspots necessary for ultrasensitive optical detection. ${ }^{33}$ SEM images in Figure 1 (b) and (c) show Au-NP clusters before and after adsorption of $1 \mu \mathrm{M}$ insulin, respectively. Raman spectra obtained from powdered insulin (Figure 1d) and 3D Au-NP clusters incubated in $1 \mathrm{mM}$ insulin (Figure 1e) were collected as reference Raman signatures for insulin sensing and show consistent peak intensities and location. The most intense peak at $1002 \mathrm{~cm}^{-1}$ corresponding to the ring-breathing mode of aromatic phenylalanine was used to monitor the change in Raman intensity as a function of 
insulin concentration. ${ }^{37}$ Measurements were performed at the center of each chip and at four additional points -- top, bottom, left, and right, about $0.5 \mathrm{~mm}$ away from the center location -- to characterize the Raman intensity and spatial uniformity. The change in SERS signal intensity as a function of insulin concentration between $100 \mathrm{pM}$ to $50 \mathrm{nM}$ is shown in Figures 2 (a) and (b). The $1002 \mathrm{~cm}^{-1}$ peak was identifiable at $100 \mathrm{pM}$ and a linear increase in the Raman intensity was observed as the insulin concentration increased from $100 \mathrm{pM}$ to $10 \mathrm{nM}$. The Raman intensity reached saturation at insulin concentrations greater than $50 \mathrm{nM}$, which indicates an almost complete monolayer coverage. Insulin can exist in solutions as a hexamer $(\sim 5 \mathrm{~nm}$ in diameter and $3.5 \mathrm{~nm}$ in height) in the presence of zinc ions at neutral $\mathrm{pH}$, as a dimer $(\sim 4.5 \mathrm{~nm}$ diameter and $\sim 2 \mathrm{~nm}$ in height $)$ or as a monomer $(\sim 2 \mathrm{~nm}$ diameter and height $\sim 2 \mathrm{~nm}) .{ }^{38}$ As no zinc ions were added to insulin containing buffers, the molecules are expected to be in a monomer-dimer equilibrium. Given the size of insulin molecules, even if there were a second layer sitting on top of the first layer, the second layer would be too far away from the surface to significantly benefit from the near-field enhancement on the surface, and the Raman-emission contribution by the second and/or third layers would be minimal. ${ }^{39}$ The signal-to-noise ratio (SNR) at $100 \mathrm{pM}$ was calculated to be approximately 8.5 , and the theoretical detection limit was calculated to be $35 \mathrm{pM}$ for a minimum acceptable SNR of 3 .

For typical SERS substrates, even a small displacement of the incident laser could often lead to a huge change in Raman signal intensity due to non-uniformly distributed SERS hotspots. In order to improve the consistency of SERS measurements, recent focus has been placed on improving the chip-scale measurement uniformity either by implementing better substrates, ${ }^{31-34}$ or employing better measurement strategies such as areal averaging, ${ }^{35-36}$ which involves scanning a larger area on the substrate to collect and average more Raman-signal data points as opposed to 
making a single point measurement. Two-dimensional (2D) mapping of Raman spectra was performed over a $1 \times 1 \mathrm{~mm}^{2}$ area with a step size of $20 \mu \mathrm{m}$ on a chip coated with a $10-\mathrm{nM}$ insulin solution (Figure 2c). The relative standard deviation (RSD) of the signal fluctuation (calculated using five points distributed across the substrate as mentioned earlier) was $4.3 \%$, indicating uniform enhancement of Raman signal over a large area of the sensor. A histogram of the Raman signals obtained over the mapped region also reveals a very narrow intensity distribution and indicates uniform enhancement (Figure 2d). Both highly uniform 2D areal scan results (Figure 2c, d) and the saturation of the Raman-intensity curve support that the insulin coverage on the surface is a monolayer - or the major contribution comes from the monolayer, and the transport mechanism to the surface is a relatively uniform diffusion process. Areal averaging was also explored to check if we can further improve the spatial uniformity of Raman measurements. Figure 2e shows the RSD as a function of the scan area used for averaging. The results show that increasing the averaging scan area improves the measurement uniformity. For example, increasing the average scan area from $50 \times 50 \mu \mathrm{m}^{2}$ to $400 \times 400 \mu \mathrm{m}^{2}$ reduces the RSD from $3.6 \%$ to $1.7 \%$. However, it should be noted the improvement in measurement uniformity from areal averaging is small, only about $1-3 \%$, which is a testament to the excellent spatial uniformity of signal obtained from the 3D Au-NP substrate. This excellent spatial uniformity of SERS measurements also indicates a complete and uniform coverage of insulin on the substrate.

In order to demonstrate a practical, rapid detection of low-level insulin using the SERS substrate, a $200 \mu \mathrm{L}$ drop of 100-pM insulin solution was applied to the surface of fresh SERS chips and dried by placing on a hotplate (without stirring) at $50^{\circ} \mathrm{C}$ for 30 minutes. The locations and intensities of the Raman peaks measured from the evaporation-prepared samples (Figure 2f) matched the substrates incubated in insulin solutions for 12 hours. The droplet is typically $0.5 \mathrm{~cm}$ 
in diameter, and signal was collected from central regions of the placed droplet, where insulin transport should be governed by diffusion and any thermal gradient-derived convective currents, thus avoiding the edges where evaporation-driven concentrating ring effects are observed. As mentioned earlier, even if more than one layer of molecules were assembled on the surface, the second layer would be too far away from the surface to benefit from the near-field enhancement on the surface as the Raman-emission contribution by the second and/or third layers would be minimal. ${ }^{39} \mathrm{~A}<10 \%$ SNR reduction was observed in the faster evaporation-detection technique and the minimum detectable concentration was calculated to be approximately $40 \mathrm{pM}$.

From our measurements, the enhancement factor of the substrate for sensing insulin was calculated to be $3 \times 10^{7} .^{40}$ This enhancement primarily originates from the exceptionally strong plasmonic (electromagnetic) enhancement of the 3D Au-NP clusters. Capture of insulin on the gold substrate can be mediated through two possible interactions: a) hydrophobic regions of the insulin molecules can promote adsorption onto the substrate, which exhibits extreme hydrophobicity with a measured water-substrate contact angle of 140 degrees and b) through strong binding interaction between the disulphide bonds of insulin molecules and the gold atoms of the SERS substrate. ${ }^{41-43}$ The chemical interaction between sulphur and gold atoms is well known and has been extensively utilized by researchers in the form of thiol-gold linkages. ${ }^{44-45}$ These interactions between the insulin molecules and gold substrate can further contribute towards enhancement of Raman signal. ${ }^{46}$

We then utilized our label-free sensing method to assess the functionality of human pancreatic islets. Secretions from the islets were collected using a flow-culture system under low glucose $(3 \mathrm{mM})$ and high glucose $(17 \mathrm{mM})$ environments as discussed in the experimental section (Supporting information file). ${ }^{8}$ We established the insulin concentrations of the collected 
secretion samples by performing the ELISA on an aliquot extracted from each secretion sample. We applied the collected secretion samples to 3D Au-NP cluster chips and measured SERS signals (Figure 3). Difference in the SERS intensity from low-glucose and high-glucose secretions was clearly visible. Then we compared the SERS signals of the islet secretions with those obtained previously in the calibration test performed on insulin in PBS buffer. A regression analysis was carried out on the islet-secretion measurements by fitting a line with the slope obtained from the calibration test (Figure 3b). While the slopes are the same, the $y$-intercept of the line fitted to the islet-secretion measurements (Figure $3 b, y$-intercept $=74.8$ ) is approximately six times larger than that of the line fitted to the calibration-test results (inset of Figure 2b, $y$ intercept $=12.6$ ), indicating that the line was shifted upward for the islet case. This was due to the presence of other molecules (primarily HSA) in the islet secretion buffer, leading to an increase in the background intensity bias that pushed up the overall spectral intensity. Other than the difference in the background intensity bias, our islet-secretion measurements were highly consistent with the calibration measurements made using insulin in PBS. In the islet-secretion measurements, the Raman intensity of the insulin peak at $1002 \mathrm{~cm}^{-1}$ consistently increased as a function of increasing glucose and insulin concentrations (Figure S2). The islet measurements also showed a highly linear relationship with the ELISA measurements as shown in Figure 3b, and this linear relationship was well described by a line whose slope is the same as that obtained in the calibration test. The coefficient of determination $\left(\mathrm{R}^{2}\right)$ was 0.97 , indicating an excellent linear fit (Figure 3b).

The RSDs for the Raman signals from islet-secretion samples were calculated as $5 \%$ and $5.3 \%$ for the low- and high-glucose samples, respectively, and showed a small increase as compared to $4.3 \%$ obtained from calibration-test samples (Figure 2e). There was no significant 
change in the calculated detection limit for the islet secretions, which was estimated at approximately $36 \mathrm{pM}$ using a SNR threshold of 3. Although the constant bias of the background signal (indicated by the elevated $y$-intercept in Figure $3 b$ as compared to $y$-intercept in Figure $2 b$ (inset)) increased by a factor of six, the mean noise level (after subtracting the background bias) remained almost the same as in the islet-measurement case. As a result, the SNR remains almost constant from the insulin-in-PBS case to the islet case. And, the slope of the Raman-intensity vs. insulin concentrations produced using our approach remained the same in both cases as shown in Fig. $3 b$ even if the background bias increased in the islet case. The detection limit in this case signifies the minimum detectable change in insulin concentration in the target concentration range, which is important to characterize islet performance.

The increase in the background noise (after subtracting the constant bias) in the islet case was minimal because the influence of other molecules on our measurements was very weak. As glucose has a weak Raman cross-section and poor adhesion to metals, ${ }^{47-48}$ change in glucose concentration has no influence on obtained Raman signal. The influence on Raman signals from other hormones such as glucagon, amylin, somatostatin, and pancreatic polypeptide whose concentration could change in response to elevating glucose levels was considered as a potential source of error. However, the physiological concentration of glucagon released in islet secretions is less than $17 \mathrm{pM}$, which is lower than the detection limit of the sensor, and its influence was considered insignificant. ${ }^{49-50}$ The concentration levels of amylin and somatostatin in the islet secretion are also very small, about $1 / 100$ and $1 / 25$ of the insulin concentration, respectively. ${ }^{51-52}$ And, pancreatic polypeptide does not show the Raman peak at $1002 \mathrm{~cm}^{-1}$ from phenylalanine. ${ }^{53}$ Hence, when detecting insulin in islet secretion using the peak at $1002 \mathrm{~cm}^{-1}$, the influence of other hormones on our measurements can be ignored. 


\section{Conclusion and Discussion}

Using a Raman-enhancing plasmonic platform, we have demonstrated highly sensitive optical detection of insulin at physiological and clinically-relevant concentrations. The chip-scale uniformity of the measurements is excellent, and we successfully characterized the insulin levels in the pancreatic islet secretions. To the best of our knowledge, this is the first demonstration of clinically-relevant, spatially uniform protein sensing on-chip using a highly enhancing nonresonant SERS substrate, whereas other reports have achieved highly uniform and sensitive detection only with smaller molecules such as Rhodamine $6 \mathrm{G} .{ }^{31-34}$ Based on surface interactions, other gold-based SERS surfaces should elicit similar adsorption behavior and allow detection of insulin in principle although the resulting accuracy and sensitivity of qualitative sensing may vary from substrate to substrate. The limit of detection far exceeds previously reported label-free optical sensing-based results and can be further improved by using molecule-concentrating techniques and post-measurement signal processing. ${ }^{54-56}$ The work presented in this manuscript can directly lead to development of convenient label-free optical sensors for large-scale on-chip screening of pancreatic islets required for islet transplantation therapy. The future direction will also be aimed at improving the specificity of hormone detection using these substrates in media such as blood. Techniques such as complex spectrum analysis including weighted analysis of multiple peaks, as well as application of small and cost-effective linkers such as aptamers with similar or better affinity for insulin as existing antibodies need to be developed towards this goal. These advancements can lead to cost-effective, easy-to-use, and sufficiently accurate insulin sensors, not yet commercially available, for measurements at home, laboratories and clinics. 
Acknowledgments: This work was supported by the Caltech-City-of-Hope Collaboration Fund, Heritage Medical Research Institute, and the Samsung Grand Research Opportunity.

Notes: The authors declare no competing financial interest.

Supporting Information: Supporting information is available free of charge on the ACS publications website. Experimental Section discussing the methods used for fabrication of the 3D Au-NP substrate and insulin sensing are discussed.

\section{Figures}

a

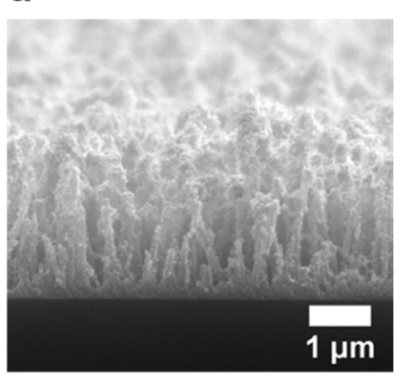

d

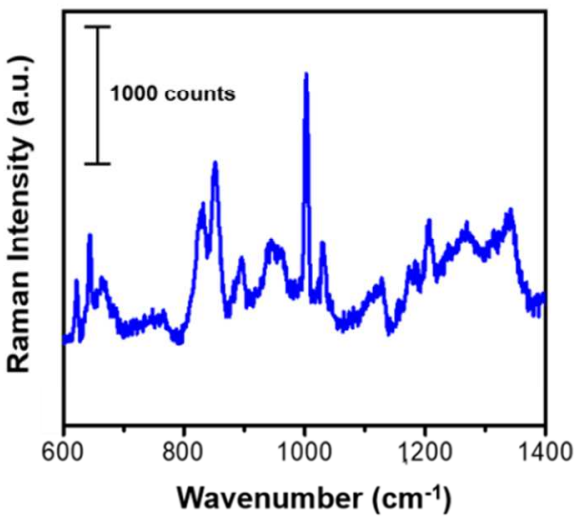

b

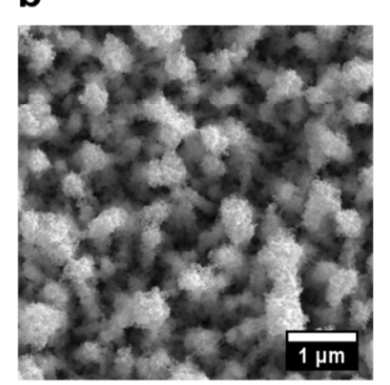

e

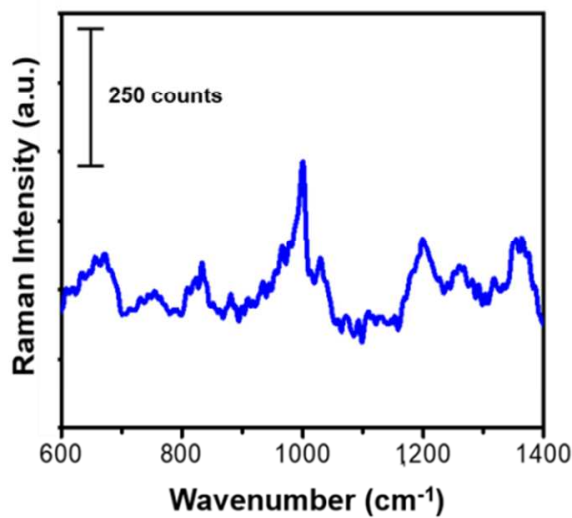

Figure 1: (a) Cross-sectional SEM image showing the vertical pillar-like 3D arrangement of the Au-NP clusters on the substrate. (b) SEM of a substrate before and (c) after insulin incubation 
(top view). (d) Raman spectra of human insulin powder and (e) a SERS substrate incubated in 1 $\mathrm{mM}$ insulin solution. The most intense peak at $1002 \mathrm{~cm}^{-1}$ corresponds to the ring-breathing mode of aromatic phenylalanine and was used to monitor changes in Raman intensity as a function of insulin concentration.

a

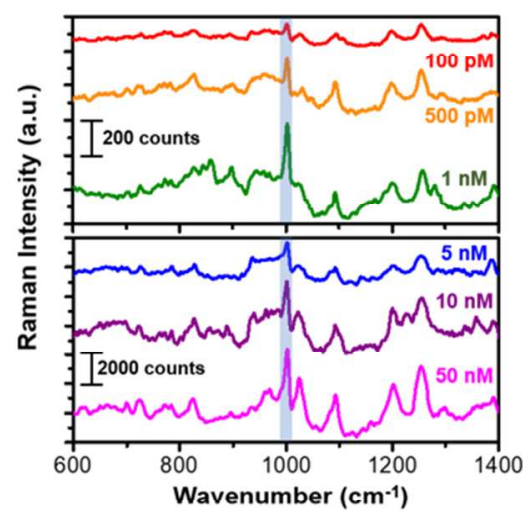

C

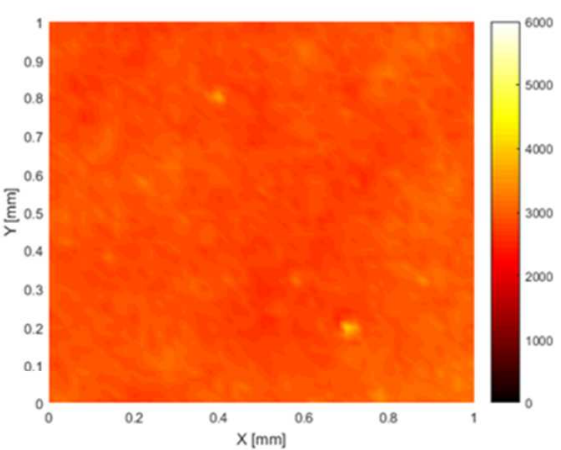

e

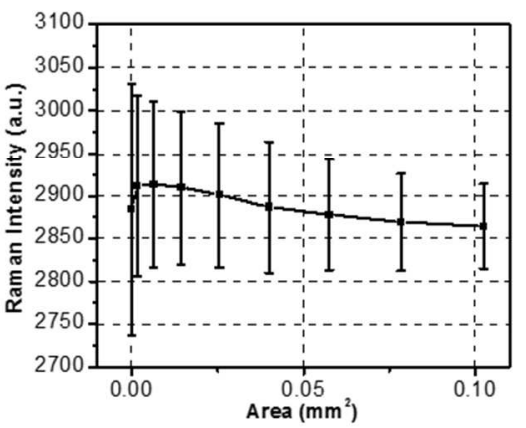

b

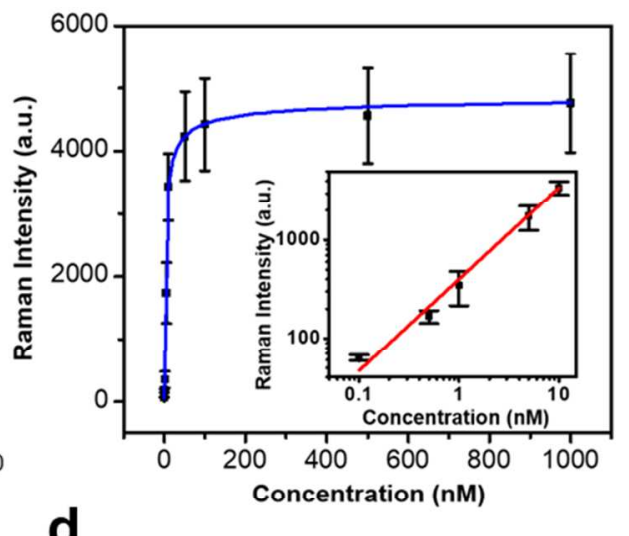

d

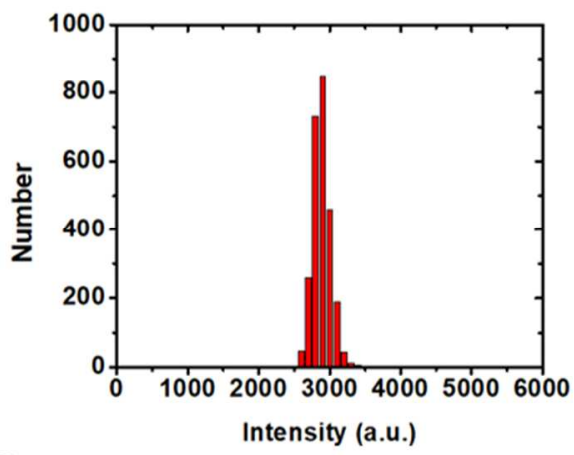

f

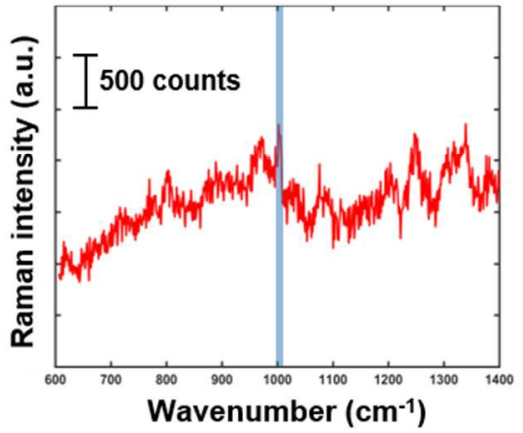


Figure 2: (a) SERS spectra of $100 \mathrm{pM}, 500 \mathrm{pM}, 1 \mathrm{nM}, 5 \mathrm{nM}, 10 \mathrm{nM}, 50 \mathrm{nM}$ human insulin solutions highlighting the target peak at $1002 \mathrm{~cm}^{-1}$. (b) Experimentally measured relationship between the insulin concentration and SERS intensity; the inset shows a linear relationship between the Raman intensity and concentration from $100 \mathrm{pM}$ to $10 \mathrm{nM}$. (c) 2D Raman-intensity mapping of a SERS chip $\left(1 \times 1 \mathrm{~mm}^{2}\right)$ after incubating in a $10 \mathrm{nM}$ insulin solution. The RSD of the Raman signal over the $1 \times 1 \mathrm{~mm}^{2}$ area was found to be $4.3 \%$, demonstrating excellent spatial uniformity. (d) Histogram showing the narrow distribution of Raman intensity over the mapped region. (e) Graph showing the mean and standard deviation of Raman signals collected from the sample as a function of the scan size used for areal averaging. The relative standard deviation could be further improved down to $1.7 \%$ using areal averaging. (f) A SERS spectrum captured from a 3D Au-NP SERS chip coated with a drop of 100-pM human insulin solution followed by rapid on-chip evaporation. 


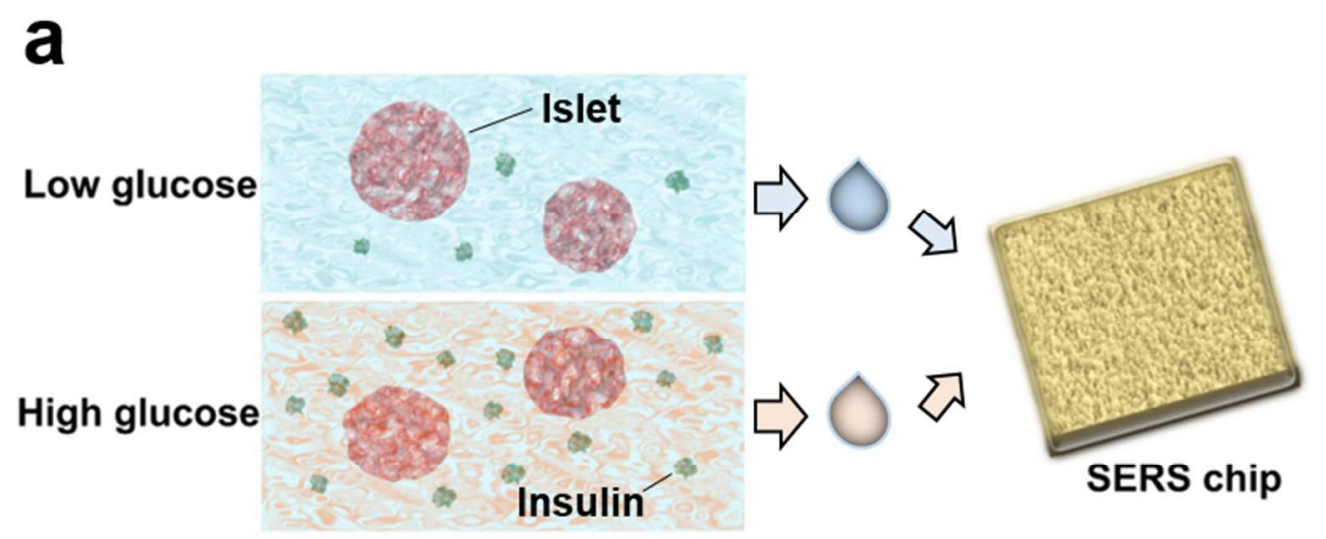

b

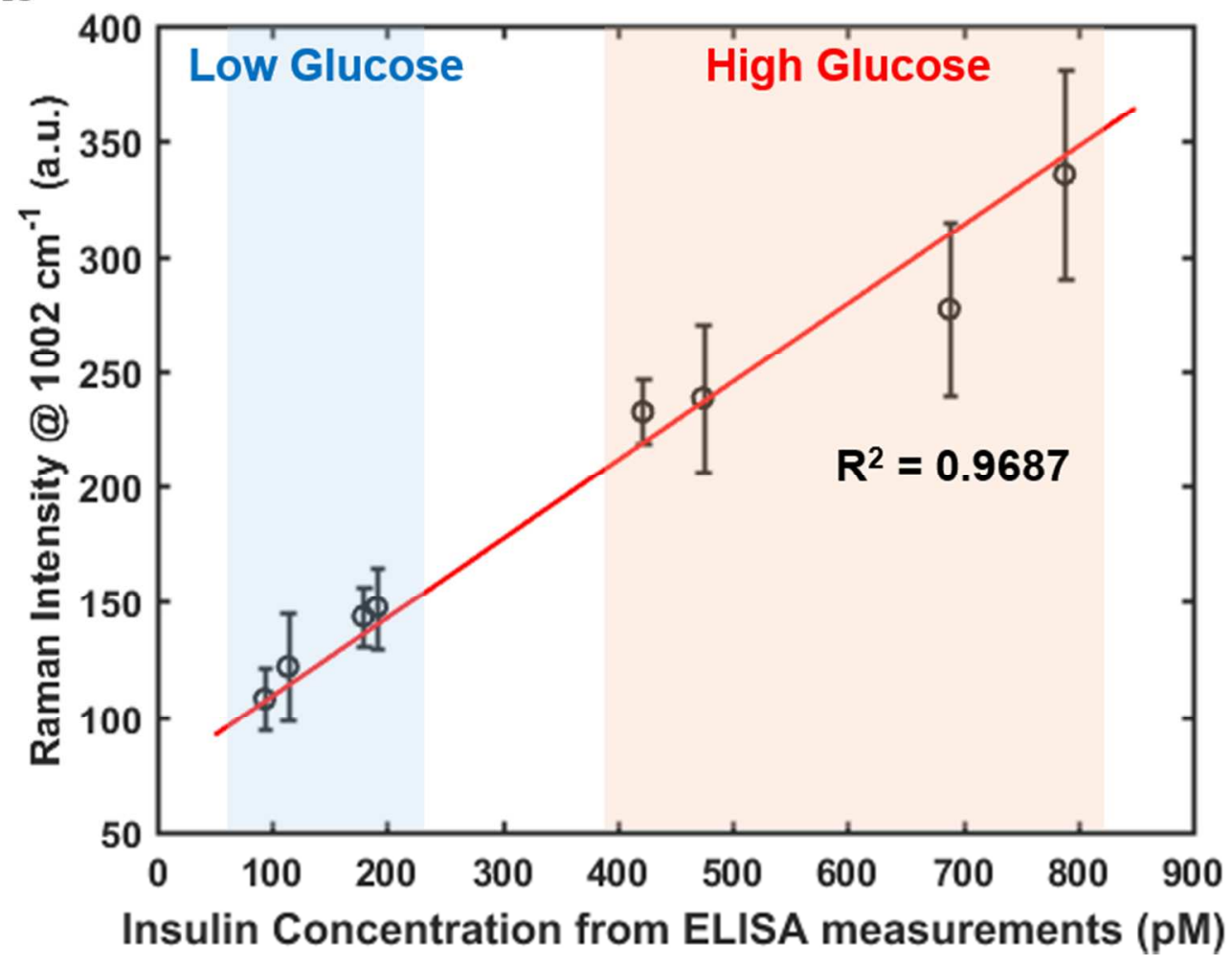

Figure 3: a) Illustration of using a 3D Au-NP SERS chip for analysis of pancreatic islet secretions. Secretions from islets were obtained under low and high glucose conditions. Insulin concentrations in these secretions were first measured using ELISA. The secretion samples were then added to SERS substrates to measure the Raman spectra and intensity. b) Raman signal intensities measured on various islet secretions (the vertical axis) correlated with the concentrations of insulin in the samples obtained using the ELISA method (the horizontal axis). 
The red line whose slope is the same as that obtained in the calibration test (inset of Figure 2(b)) exhibited the coefficient of determination $\left(\mathrm{R}^{2}\right)$ close to 1 , indicating an excellent fit. 


\section{References}

1. Seltzer, H. S.; Allen, E. W.; Herron Jr, A. L.; Brennan, M. T., Insulin secretion in response to glycemic stimulus: relation of delayed initial release to carbohydrate intolerance in mild diabetes mellitus. J. Clin. Invest. 1967, 46, 323-335.

2. Weyer, C.; Bogardus, C.; Mott, D. M.; Pratley, R. E., The natural history of insulin secretory dysfunction and insulin resistance in the pathogenesis of type 2 diabetes mellitus. J. Clin. Invest. 1999, 104, 787-794.

3. Yalow, R. S.; Berson, S. A., Plasma insulin concentrations in nondiabetic and early diabetic subjects: determinations by a new sensitive immuno-assay technic. Diabetes 1960, 9, 254-260.

4. Melmed, S.; Polonsky, K. S.; Larsen, P. R.; Kronenberg, H. M., Williams textbook of endocrinology. $12^{\text {th }}$ ed.; Elsevier Saunders: Philadelphia, 2011.

5. Shapiro, A. J.; Lakey, J. R.; Ryan, E. A.; Korbutt, G. S.; Toth, E.; Warnock, G. L.; Kneteman, N. M.; Rajotte, R. V., Islet transplantation in seven patients with type 1 diabetes mellitus using a glucocorticoid-free immunosuppressive regimen. N. Engl. J. Med. 2000, 343, 230-238.

6. $\quad$ Barton, F. B.; Rickels, M. R.; Alejandro, R.; Hering, B. J.; Wease, S.; Naziruddin, B.; Oberholzer, J.; Odorico, J. S.; Garfinkel, M. R.; Levy, M., Improvement in outcomes of clinical islet transplantation: 1999-2010. Diabetes Care 2012, 35, 1436-1445.

7. Nano, R.; Clissi, B.; Melzi, R.; Calori, G.; Maffi, P.; Antonioli, B.; Marzorati, S.; Aldrighetti, L.; Freschi, M.; Grochowiecki, T., Islet isolation for allotransplantation: variables associated with successful islet yield and graft function. Diabetologia 2005, 48, 906-912.

8. $\quad$ Sweet, I.; Gilbert, M.; Scott, S.; Todorov, I.; Jensen, R.; Nair, I.; Al $\square$ Abdullah, I.; Rawson, J.; Kandeel, F.; Ferreri, K., Glucose $\square$ stimulated increment in oxygen consumption rate as a standardized test of human islet quality. Am. J. Transplant. 2008, 8, 183-192.

9. Papas, K. K.; Suszynski, T. M.; Colton, C. K., Islet assessment for transplantation. Curr. Opin. Organ Transplant. 2009, 14, 674-682. 
10. Mork, T. A.; Killeen, C. T.; Patel, N. K.; Dohnal, J. M.; Karydes, H. C.; Leikin, J. B., Massive insulin overdose managed by monitoring daily insulin levels. Am. J. Ther. 2011, 18, e162-e166.

11. Andersen, L.; Dinesen, B.; Jørgensen, P.; Poulsen, F.; Røder, M., Enzyme immunoassay for intact human insulin in serum or plasma. Clin. Chem. 1993, 39, 578-582.

12. Zhang, X.; Zhu, S.; Deng, C.; Zhang, X., An aptamer based on-plate microarray for highthroughput insulin detection by MALDI-TOF MS. Chem. Commun. 2012, 48, 2689-2691.

13. Cha, T.-G.; Baker, B. A.; Sauffer, M. D.; Salgado, J.; Jaroch, D.; Rickus, J. L.; Porterfield, D. M.; Choi, J. H., Optical nanosensor architecture for cell-signaling molecules using DNA aptamercoated carbon nanotubes. ACS Nano 2011, 5, 4236-4244.

14. Xu, M.; Luo, X.; Davis, J. J., The label free picomolar detection of insulin in blood serum. Biosens. Bioelectron. 2013, 39, 21-25.

15. Roper, M. G.; Shackman, J. G.; Dahlgren, G. M.; Kennedy, R. T., Microfluidic chip for continuous monitoring of hormone secretion from live cells using an electrophoresis-based immunoassay. Anal. Chem. 2003, 75, 4711-4717.

16. Guillo, C.; Roper, M. G., Two-color electrophoretic immunoassay for simultaneous measurement of insulin and glucagon content in islets of Langerhans. Electrophoresis 2008, 29, 410-416.

17. Gobi, K. V.; Iwasaka, H.; Miura, N., Self-assembled PEG monolayer based SPR immunosensor for label-free detection of insulin. Biosens. Bioelectron. 2007, 22, 1382-1389.

18. Wang, Y.; Gao, D.; Zhang, P.; Gong, P.; Chen, C.; Gao, G.; Cai, L., A near infrared fluorescence resonance energy transfer based aptamer biosensor for insulin detection in human plasma. Chem. Commun. 2014, 50, 811-813.

19. Luo, X.; Xu, M.; Freeman, C.; James, T.; Davis, J. J., Ultrasensitive label free electrical detection of insulin in neat blood serum. Anal. Chem. 2013, 85, 4129-4134.

20. Schnell, U.; Dijk, F.; Sjollema, K. A.; Giepmans, B. N., Immunolabeling artifacts and the need for live-cell imaging. Nat. Methods 2012, 9, 152-158. 
21. Blasi, T.; Hennig, H.; Summers, H. D.; Theis, F. J.; Cerveira, J.; Patterson, J. O.; Davies, D.; Filby, A.; Carpenter, A. E.; Rees, P., Label-free cell cycle analysis for high-throughput imaging flow cytometry. Nat. Commun. 2016, 7, 10256.

22. Fleischmann, M.; Hendra, P. J.; McQuillan, A. J., Raman spectra of pyridine adsorbed at a silver electrode. Chem. Phys. Lett. 1974, 26, 163-166.

23. Albrecht, M. G.; Creighton, J. A., Anomalously intense Raman spectra of pyridine at a silver electrode. J. Am. Chem. Soc. 1977, 99, 5215-5217.

24. Nie, S. M.; Emery, S. R., Probing single molecules and single nanoparticles by surface-enhanced Raman scattering. Science 1997, 275, 1102-1106.

25. Kneipp, K.; Wang, Y.; Kneipp, H.; Perelman, L. T.; Itzkan, I.; Dasari, R. R.; Feld, M. S., Single molecule detection using surface-enhanced Raman scattering (SERS). Phys. Rev. Lett. 1997, 78, 1667.

26. Bantz, K. C.; Meyer, A. F.; Wittenberg, N. J.; Im, H.; Kurtulus, O.; Lee, S. H.; Lindquist, N. C.; Oh, S.-H.; Haynes, C. L., Recent progress in SERS biosensing. Phys. Chem. Chem. Phys. 2011, $13,11551-11567$.

27. Drachev, V. P.; Thoreson, M. D.; Khaliullin, E. N.; Davisson, V. J.; Shalaev, V. M., Surfaceenhanced Raman difference between human insulin and insulin lispro detected with adaptive nanostructures. J. Phys. Chem. B 2004, 108, 18046-18052.

28. Drachev, V. P.; Thoreson, M. D.; Nashine, V.; Khaliullin, E. N.; Ben-Amotz, D.; Davisson, V. J.; Shalaev, V. M., Adaptive silver films for surface-enhanced Raman spectroscopy of biomolecules. J. Raman Spectrosc. 2005, 36, 648-656.

29. Keskin, S.; Kahraman, M.; Çulha, M., Differential separation of protein mixtures using convective assembly and label-free detection with surface enhanced Raman scattering. Chem. Commun. 2011, 47, 3424-3426. 
30. Matteini, P.; de Angelis, M.; Ulivi, L.; Centi, S.; Pini, R., Concave gold nanocube assemblies as nanotraps for surface-enhanced Raman scattering-based detection of proteins. Nanoscale 2015, 7, 3474-3480.

31. Kanipe, K. N.; Chidester, P. P.; Stucky, G. D.; Moskovits, M., Large Format Surface-Enhanced Raman Spectroscopy Substrate Optimized for Enhancement and Uniformity. ACS Nano 2016, 10, 7566-7571.

32. Lin, D.; Wu, Z.; Li, S.; Zhao, W.; Ma, C.; Wang, J.; Jiang, Z.; Zhong, Z.; Zheng, Y.; Yang, X., Large-Area Au-Nanoparticle-Functionalized Si Nanorod Arrays for Spatially Uniform SurfaceEnhanced Raman Spectroscopy. ACS Nano 2017, 11, 1478-1487.

33. Yang, D.; Cho, H.; Koo, S.; Vaidyanathan, S. R.; Woo, K.; Yoon, Y.; Choo, H., Simple, LargeScale Fabrication of Uniform Raman-Enhancing Substrate with Enhancement Saturation. ACS Appl. Mater. Interfaces 2017, 9, 19092-19101.

34. Hoang, P.; Khashab, N. M., Non-Resonant Large Format Surface Enhanced Raman Scattering Substrates for Selective Detection and Quantification of Xylene Isomers. Chem. Mater. 2017, 29, 1994-1998.

35. Lee, M.; Lee, S.; Lee, J.-h.; Lim, H.-w.; Seong, G. H.; Lee, E. K.; Chang, S.-I.; Oh, C. H.; Choo, J., Highly reproducible immunoassay of cancer markers on a gold-patterned microarray chip using surface-enhanced Raman scattering imaging. Biosens. Bioelectron. 2011, 26, 2135-2141.

36. Chang, H.; Kang, H.; Ko, E.; Jun, B.-H.; Lee, H.-Y.; Lee, Y.-S.; Jeong, D. H., PSA Detection with Femtomolar Sensitivity and a Broad Dynamic Range Using SERS Nanoprobes and an AreaScanning Method. ACS Sens. 2016, 1, 645-649.

37. Ortiz, C.; Zhang, D.; Xie, Y.; Davisson, V. J.; Ben-Amotz, D., Identification of insulin variants using Raman spectroscopy. Anal. Biochem. 2004, 332, 245-252.

38. Blundell, T.; Cutfield, J.; Cutfield, S.; Dodson, E.; Dodson, G.; Hodgkin, D.; Mercola, D., Threedimensional atomic structure of insulin and its relationship to activity. Diabetes 1972, 21, 492 505. 
42. Lacerda, S. H. D. P.; Park, J. J.; Meuse, C.; Pristinski, D.; Becker, M. L.; Karim, A.; Douglas, J. F., Interaction of gold nanoparticles with common human blood proteins. ACS Nano 2009, 4, 365-379.

43. Welinder, A. C.; Zhang, J.; Steensgaard, D. B.; Ulstrup, J., Adsorption of human insulin on single-crystal gold surfaces investigated by in situ scanning tunnelling microscopy and electrochemistry. Phys. Chem. Chem. Phys. 2010, 12, 9999-10011.

44. Bain, C. D.; Troughton, E. B.; Tao, Y. T.; Evall, J.; Whitesides, G. M.; Nuzzo, R. G., Formation of monolayer films by the spontaneous assembly of organic thiols from solution onto gold. J. Am. Chem. Soc. 1989, 111, 321-335.

45. Bain, C. D.; Evall, J.; Whitesides, G. M., Formation of monolayers by the coadsorption of thiols on gold: variation in the head group, tail group, and solvent. J. Am. Chem. Soc. 1989, 111, $7155-$ 7164.

46. Campion, A.; Ivanecky III, J.; Child, C.; Foster, M., On the mechanism of chemical enhancement in surface-enhanced Raman scattering. J. Am. Chem. Soc. 1995, 117, 11807-11808.

47. McCreery; L., R., Raman Spectroscopy for Chemical Analysis. Wiley-Interscience: New York, 2000.

48. Shafer-Peltier, K.; Haynes, C.; Glucksberg, M.; Van Duyne, R., Toward a glucose biosensor based on surface-enhanced Raman scattering. J. Am. Chem. Soc. 2003, 125, 588-593. 
49. Alford, F.; Bloom, S.; Nabarro, J., Glucagon levels in normal and diabetic subjects: use of a specific immunoabsorbent for glucagon radioimmunoassay. Diabetologia 1977, 13, 1-6.

50. Gardner, D.; Shoback, D., Appendix: Normal Hormone Reference Ranges. Greenspan's Basic \& Clinical Endocrinology. McGraw-Hill: New York, 2011.

51. Adeghate, E.; Kalász, H., Amylin Analogues in the Treatment of Diabetes Mellitus: Medicinal Chemistry and Structural Basis of its Function. Open Med. Chem. J. 2011, 5, 78-81.

52. Sorenson, R. L.; Lindell, D. V.; Elde, R. P., Glucose stimulation of somatostatin and insulin release from the isolated, perfused rat pancreas. Diabetes 1980, 29, 747-751.

53. Hilderink, J.; Otto, C.; Slump, C.; Lenferink, A.; Engelse, M.; van Blitterswijk, C.; de Koning, E.; Karperien, M.; van Apeldoorn, A., Label-free detection of insulin and glucagon within human islets of Langerhans using Raman spectroscopy. PLoS One 2013, 8, e78148.

54. Barik, A.; Otto, L. M.; Yoo, D.; Jose, J.; Johnson, T. W.; Oh, S.-H., Dielectrophoresis-enhanced plasmonic sensing with gold nanohole arrays. Nano Lett. 2014, 14, 2006-2012.

55. Kumar, S.; Cherukulappurath, S.; Johnson, T. W.; Oh, S.-H., Millimeter-sized suspended plasmonic nanohole arrays for surface-tension-driven flow-through SERS. Chem. Mater. 2014, 26, 6523-6530.

56. Kumar, S.; Johnson, T. W.; Wood, C. K.; Qu, T.; Wittenberg, N. J.; Otto, L. M.; Shaver, J.; Long, N. J.; Victora, R. H.; Edel, J. B., Oh, S.-H. Template-Stripped Multifunctional Wedge and Pyramid Arrays for Magnetic Nanofocusing and Optical Sensing. ACS Appl. Mater. Interfaces 2016, 8, 9319-9326. 
For TOC only:
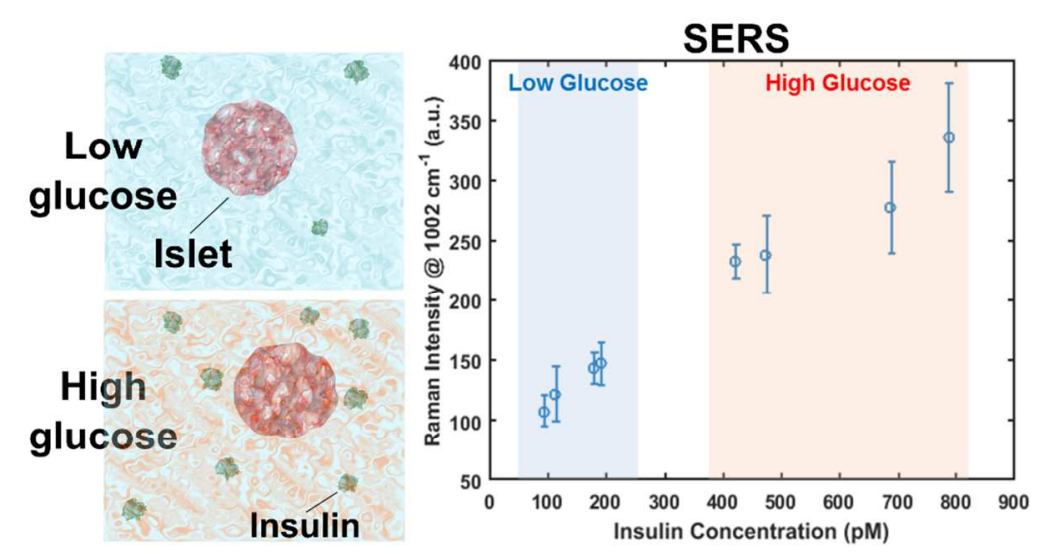